\title{
Evaluating Resident Training in Oculoplastic Surgery: A Case Series of 104 Eviscerations
}

\author{
Brittany A. Simmons, MD ${ }^{1}$ Anna Artymowicz, BS ${ }^{2}$ Mithra O. Gonzalez, MD ${ }^{1,3,4}$ \\ ${ }^{1}$ Department of Ophthalmology, Flaum Eye Institute, University of \\ Rochester, Rochester, New York \\ 2 Department of Graduate Medical Education, University of Rochester \\ School of Medicine and Dentistry, Rochester, New York \\ ${ }^{3}$ Department of Oral and Maxillofacial Surgery, Eastman Institute of \\ Oral Health, University of Rochester, Rochester, New York \\ ${ }^{4}$ Department of Otolaryngology, University of Rochester, Rochester, \\ New York \\ J Acad Ophthalmol 2018;10:e108-e113.

\begin{abstract}
Address for correspondence Brittany A. Simmons, MD, Department of Ophthalmology, Flaum Eye Institute, University of Rochester, 601 Elmwood Avenue, Rochester, NY 14642 (e-mail: Brittany_Simmons@URMC.Rochester.edu).
\end{abstract}

\begin{abstract}
Keywords

- anophthalmic surgery

- ocular evisceration

- ophthalmology resident performance

- oculoplastic training

- ophthalmology resident outcomes

Purpose The aim of this study is to evaluate resident surgical performance based on complications after ocular evisceration.

Methods A retrospective chart review of eviscerations performed between October 2011 and May 2017 by ophthalmology residents as the primary or assistant surgeon under the guidance of a single oculofacial plastic surgeon (M.O.G.) was completed. Data collected included reason for evisceration, resident participation in the case and resident's month of oculoplastic training, surgical technique, subsequent complications, and duration of follow-up.

Results There were no significant differences in complication rates or surgical sequelae in resident-led versus attending-led surgeries. The complication rate for all cases in total was 5.77\%. A slight negative correlation existed between the resident's month of training and the presence of postoperative complications. The number of adverse events was found to be significantly correlated with the duration of patient follow-up.

Discussion Ocular eviscerations performed by ophthalmology residents as primary surgeons achieve outcomes equivalent to published reports, suggesting ocular eviscerations are a safe, effective procedure wherein residents can refine surgical skills. Some surgical sequelae may be linked to particular surgeons, implying evisceration outcomes can be used to assess resident surgical performance. Fewer adverse events arose as the resident's length of oculoplastic training increased, but this finding did not reach significance. Larger studies are needed to explore these trends.
\end{abstract}

Evisceration is the removal of the intraocular contents of the eye, leaving behind a scleral shell with preserved extraocular tissues. Evisceration is performed for several reasons, including trauma, phthisis bulbi, cosmesis, and blind, painful eyes. Compared with eye removal procedures like enucleation and exenteration, evisceration is considered technically easier, preserves more tissue, offers improved implant motility, and boasts a better cosmetic result. ${ }^{1-7}$ Recent studies also suggest that eviscerations have a lower complication rate regarding postoperative infection and implant extrusion. ${ }^{2,8,9}$ Historically, evisceration was avoided due to concerns for an increased risk of sympathetic ophthalmia; however, recent received

January 30, 2018 accepted after revision June 5, 2018
DOI https://doi.org/

10.1055/s-0038-1667050. ISSN 2475-4757.
Copyright $\odot 2018$ by Thieme Medical

Publishers, Inc., 333 Seventh Avenue, New York, NY 10001, USA. Tel: +1(212) 584-4662.
License terms

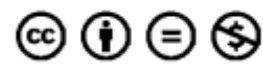


literature suggests this risk is no greater than other eye removal procedures like enucleation. ${ }^{4,8,10}$ For these reasons, the frequency of ocular evisceration has increased over the years. ${ }^{11,12}$ Typical complications of evisceration include infection and wound dehiscence with implant exposure or extrusion, as well as minor surgical sequelae such as eyelid malposition, superior sulcus deformity, enophthalmos, and cyst formation. ${ }^{7,13-21}$ Current literature explores various surgical techniques, efforts to minimize complications, and long-term outcomes of anophthalmic sockets. Many of these published works arise from training institutions. However, there is a dearth of information regarding ophthalmology trainee performance and long-term outcomes in cases of ocular evisceration. As an increasingly common, technically easier procedure in a non-seeing eye, eviscerations are ideal cases for trainees. The aim of this study is to use patient outcomes and complications to evaluate the appropriateness of ophthalmology residents performing ocular evisceration, as well as using ocular evisceration procedures to enhance surgical training and evaluate resident surgical performance.

\section{Methods}

\section{Data Acquisition}

A retrospective chart review of eviscerations performed between October 2011 and May 2017 by ophthalmology residents as the primary or assistant surgeon under the guidance of a single oculofacial plastic surgeon was completed. Data collected included reason for evisceration, resident's role in the case and resident's month of oculoplastic training, surgical technique, size of implant, subsequent complications, and duration of follow-up.

\section{Surgical Technique}

On the day of surgery, the patient was placed under general anesthesia. Phenylephrine ophthalmic drops (10\%) were applied to the operative eye, and the nonoperative eye was shielded. A 360-degree peritomy was performed, followed by conjunctival and Tenon's dissection into the quadrants at the $2,4,8$, and 10 o'clock positions. The cornea was excised and the intraocular contents were removed. In all cases, the cornea and intraocular contents were submitted for histopathologic analysis. The internal scleral surface was scrubbed repeatedly with $100 \%$ alcohol to remove any uveal antigens, followed by copious irrigation with antibiotic solution. Four scleral petals were created from the limbus to the optic nerve at the $2,4,8$, and 10 o'clock positions. An orbital sizer was used to determine an implant size that allowed for filling of the anophthalmic socket without placing undue tension when closing the scleral petals around the implant. The implant-a porous polyethylene spherical orbital implant (Medpor: Stryker, Kalamazoo, Michigan)was soaked in a combination antibiotic and anesthetic solution, and was then placed into the scleral cavity. The superior and inferior scleral petals were closed with a combination of running and interrupted slow-absorbing sutures. The horizontal petals were similarly closed. Copious irrigation was again performed with antibiotic solution to allow for visua- lization of Tenon's. In a portion of cases, slow-absorbing suture was used to close Tenon's in a running manner, followed by a separate closure of the conjunctiva with running absorbable suture. In the remainder of cases, Tenon's and conjunctiva were closed simultaneously with running absorbable suture. A combination antibiotic and steroid ointment was applied, an appropriately sized conformer was inserted, and a temporary tarsorrhaphy was placed using nonabsorbable suture. At the conclusion of the case, a retrobulbar injection of $0.75 \%$ bupivacaine with epinephrine $(1: 200,000)$ was administered, and a pressure patch and eye shield were placed. The tarsorrhaphy, pressure patch, and shield remained in place for 1 week, after which a combination antibiotic and steroid ointment was applied to the eye three times per day for the next 2 weeks.

\section{Teaching Methodology}

A single attending oculoplastic surgeon oversaw these cases. Early in the chart series, third-year residents were intermittently assigned to oculoplastic surgeries and taught in a modified "see one, do one" manner under direct supervision of the attending oculoplastic physician. Later in the chart review period, first-year residents were assigned to a 3month intensive block of oculoplastic education, including time in clinic, the minor procedure room, and the operating room. In each learning environment, a baseline level of competency was established through previous interaction and direct evaluation of surgical technique in both wet laboratory and the operating room by the attending oculoplastic surgeon. Intraoperatively, a graduated, step-wise approach to mastering the procedure was employed. If a step was completed incorrectly, the resident was informed and tasked with repeating the step correctly on the spot under demonstrative guidance of the attending surgeon until the resident was able to perform more than $50 \%$ of the key steps of the procedure, at which point the case became a primary resident case.

\section{Statistical Analysis}

Data were collected and cross-checked for errors. Percentage, range, and average mean were calculated. Statistical analysis included chi-square test, Pearson's product-moment correlation coefficient, and odds ratio (OR) calculations. Significance was determined by $p<0.05$.

\section{Results}

A total of 104 eviscerations were completed: 57 (54.8\%) were completed with a resident as the primary surgeon, while 47 (45.2\%) were completed with a resident as the assistant surgeon. Patient demographics were wide ranging (- Table 1). There were many similarities between attending and residentled case demographics; however, attending cases had nearly twice the amount of patients with penetrating ocular trauma compared with resident cases. Reasons for evisceration included blind, painful eyes from multiple etiologies (such as end-stage glaucoma; 58.7\%), endophthalmitis (12.5\%), corneal perforation (10.6\%), phthisis (9.6\%), and 
Table 1 Patient demographics

\begin{tabular}{|c|c|c|c|}
\hline Demographic & Total (\%) & Attending & Resident \\
\hline \multicolumn{4}{|l|}{ Sex } \\
\hline Female & $37(35.6 \%)$ & $16(34.0 \%)$ & $21(36.8 \%)$ \\
\hline Male & $67(64.4 \%)$ & $31(66.0 \%)$ & $36(63.2 \%)$ \\
\hline \multicolumn{4}{|l|}{ Age } \\
\hline Range & $10-97 y$ & $14-92$ y & $10-97 y$ \\
\hline Average & $60.2 \mathrm{y}$ & $44.8 \mathrm{y}$ & 58.4 y \\
\hline \multicolumn{4}{|l|}{ Laterality } \\
\hline Right eye & $42(40.4 \%)$ & $24(51.1 \%)$ & $18(31.6 \%)$ \\
\hline Left eye & $62(59.6 \%)$ & $23(48.9 \%)$ & $39(68.4 \%)$ \\
\hline \multicolumn{4}{|l|}{ Follow-up } \\
\hline Shortest & $9 d$ & $9 d$ & $9 \mathrm{~d}$ \\
\hline Longest & $53 \mathrm{mo}$ & $53 \mathrm{mo}$ & $52 \mathrm{mo}$ \\
\hline Average & $11.3 \mathrm{mo}$ & $14.2 \mathrm{mo}$ & $9.5 \mathrm{mo}$ \\
\hline \multicolumn{4}{|l|}{ Previous ocular surgery } \\
\hline Patients (no.) & $81(77.9 \%)$ & $36(76.6 \%)$ & 45 (78.9\%) \\
\hline Surgeries (no.) & 138 & 61 & 77 \\
\hline History of penetrating trauma & $28(26.9 \%)$ & $17(36.2 \%)$ & $11(19.3 \%)$ \\
\hline History of intraocular infection & $29(27.9 \%)$ & $13(27.8 \%)$ & $16(28.1 \%)$ \\
\hline
\end{tabular}

Notes: There were more male patients undergoing ocular evisceration. The average patient age was 60 years, and there was an incidental predominance of left eye evisceration. Follow-up was widely variable, with an average follow-up period of 11.3 months. Follow-up was slightly longer at 14.2 months in attending-led cases compared with 9.5 months in resident cases. The majority of patients had previous ocular surgery, which ranged from cataract extraction and glaucoma procedures to retinal detachment repairs, posterior pole vitrectomies, and repair of ruptured globes. There was nearly two times the number of patients with penetrating trauma in attending-led cases compared with resident cases.

trauma (7.7\%; - Table 2). There were nearly double the number of etiologic cases of endophthalmitis in attending cases ( $17.0 \%$ compared with $8.8 \%$ in resident cases), and chart review of attending trauma cases revealed more globally obliterative traumas than resident cases.

Duration of follow-up ranged from 9 days to nearly 5 years, with slightly longer follow-up for attending-led cases compared with resident cases ( $\mathbf{-}$ Table $\mathbf{1}$ ). During this time, a total of six complications occurred. Complications were defined as wound dehiscence, implant exposure or extrusion, surgical site infection, and sympathetic ophthalmia. Of the six complications, one occurred in resident primary cases $(1.8 \%$ of resident primary cases) and five in resident-assist cases (10.6\% of resident-assist cases). The complication rate for all cases in total was $5.77 \%$. There were no cases of implant extrusion or sympathetic ophthalmia. Minor adverse events and long-term surgical sequelae included eyelid malposition, conjunctival cysts, pyogenic granuloma, implant migration, superior sulcus defect, and enophthalmos. Sequelae occurred with a near-equal incidence in both resident-led and attending-led cases (-Table 3). There was no incidence of implant migration, superior sulcus defect, or enophthalmos. There was no significant difference in the rate of complications $\left(x^{2}(1, N=104)=3.74, p=0.05\right)$, long-term

Table 2 Reasons for ocular evisceration

\begin{tabular}{|l|l|l|l|}
\hline Reason for evisceration & \% $(\boldsymbol{n})$ & Attending & Resident \\
\hline Blind, painful eye & $58.7(61)$ & $48.9(23)$ & $66.7(38)$ \\
\hline Endophthalmitis & $12.5(13)$ & $17.0(8)$ & $8.8(5)$ \\
\hline Corneal perforation & $10.6(11)$ & $10.6(5)$ & $10.5(6)$ \\
\hline Phthisis & $9.6(10)$ & $12.8(6)$ & $7.0(4)$ \\
\hline Trauma & $7.7(8)$ & $8.5(4)$ & $7.0(4)$ \\
\hline Other & $0.9(1)$ & $2.1(1)$ & $0.0(0)$ \\
\hline
\end{tabular}

Notes: Trauma occurred within the preceding 3 months to qualify as the reason for evisceration. Attending trauma cases tended to have more globally disruptive trauma than resident cases. Blind, painful eyes occurred from several pathologies, including end-stage glaucoma and keratitis. Evisceration is absolutely contraindicated in cases of intraocular malignancy, and there was no intraocular malignancy present on histopathologic analysis of eviscerated tissue. 
Table 3 Complications, sequelae, and surgical variables after ocular evisceration

\begin{tabular}{|l|l|l|}
\hline \multirow{2}{*}{$\begin{array}{l}\text { Number of } \\
\text { eviscerations }\end{array}$} & \multicolumn{2}{|l|}{ Resident role } \\
\cline { 2 - 3 } & $\begin{array}{l}\text { Primary } \\
\text { surgeon } \\
(\boldsymbol{n}=\mathbf{5 7})\end{array}$ & $\begin{array}{l}\text { Assistant } \\
\text { surgeon } \\
(\boldsymbol{n}=\mathbf{4 7})\end{array}$ \\
\hline Major complications & 1 & 5 \\
\hline Implant exposure & 0 & 3 \\
\hline Wound dehiscence & 1 & 1 \\
\hline Infection & 0 & 1 \\
\hline Minor surgical sequelae & 21 & 18 \\
\hline Ptosis & 6 & 5 \\
\hline Other eyelid malposition & 6 & 5 \\
\hline Conjunctival cyst & 5 & 4 \\
\hline Other & 4 & 4 \\
\hline Surgical variables & & \\
\hline Implant size (average) & 19.6 mm & 20.1 mm \\
\hline $\begin{array}{l}\text { Separate or combined } \\
\text { closure of Tenon's and } \\
\text { conjunctiva }\end{array}$ & 40 separate & 44 separate \\
\hline
\end{tabular}

Notes: As a case series of 104 ocular eviscerations, the overall complication rate was $5.77 \%$. The major complication rate was $1.8 \%$ in resident primary cases and $10.6 \%$ in resident-assist cases. Minor surgical sequelae occurred with a near-equal incidence of $36.8 \%$ in resident primary and $38.3 \%$ in resident-assist cases. Other sequelae included one case each of fornix shortening (in the setting of an initial alkali burn), persistent pain (in a patient with neuropathic pain syndrome), symblepharon formation, pedunculated benign conjunctival lesion, pyogenic granuloma (occurring twice in the same patient), and chronic discharge. No significant differences were observed among surgical variables. The orbital implant size ranged from 16 to $22 \mathrm{~mm}$, with the average size being a $19.9-\mathrm{mm}$ implant. There was no statistical difference in choice of implant size $\left(X^{2}(1, N=104)=0.86, p=0.35\right)$ or adverse events $\left(X^{2}(1, N=104)=0.09, p=0.76\right)$ between these two groups. Additionally, there was no correlation between the type of conjunctiva and Tenon's closure (whether closed separately or simultaneously) with postoperative complications $\left(x^{2}(1, N=103\right.$, due to exclusion of one patient who lacked conjunctiva) $=1.11, p=0.292$; Fisher's exact test yielded $p=0.2857$ in resident surgeries, and $p=0.2920$ in attending-led surgeries), similar to a recently published report. $^{21}$

sequelae $\left(\chi^{2}(1, N=104)=1.53, p=0.22\right)$, or total adverse events (complications of surgery and minor or long-term sequelae $\left(\chi^{2}(1, N=104)=0.01, p=0.91\right)$ between resident- and attending-led cases. An infectious etiology for evisceration was associated with a slightly increased risk of experiencing a major complication in both attending- and resident-led cases $(\mathrm{OR}=1.23)$. There was a larger risk for complications in patients who had a history of previous ocular surgery $(\mathrm{OR}=3.35$ in attending cases; the single complication in resident cases was insufficient to calculate this for resident-led surgeries).

A Pearson product-moment correlation coefficient revealed a slight negative correlation between the resident's month of training and the presence of postoperative compli- cations, though this was not significant $(r=-0.23, n=40$, $p$ [one-tailed] $=0.08$ ). There was a small but significant positive correlation between the occurrence of all adverse events (complications and long-term sequelae) and duration of follow-up $(r=0.029, n=104, p$ [one-tailed] $=0.001)$.

\section{Discussion}

Ocular evisceration is employed in patients with cosmetic, painful, infectious, or other pathologic processes that compromise ocular integrity. Historically, enucleation was the preferred anophthalmic procedure in these patients, as it was believed that enucleation had a lower risk of sympathetic ophthalmia and better postoperative pain control. $^{22-26}$ The frequency of eviscerations has increased in the past few decades, ${ }^{11,12}$ in part because evisceration is considered a technically easier procedure and now has a known low rate of sympathetic ophthalmia. ${ }^{4,8,10,27}$ As such, ocular evisceration is an ideal procedure for ophthalmology trainees. However, review of the current literature reveals a lack of information regarding ophthalmology resident performance and outcomes in cases of ocular evisceration. In this study, we found that not only were trainees capable of safely performing ocular eviscerations with a complication rate similar to published reports but also that a trainee's surgical skills could be both taught and evaluated by performance of ocular evisceration procedures and assessing postoperative complications.

Our rate of complications (1.8\% in resident primary cases and $10.6 \%$ in resident-assist cases) falls within published reports, suggesting that evisceration surgery is a safe procedure for ophthalmology residents to learn and practice surgical skills. A review of the literature shows complication rates after evisceration range from 0 to $53.8 \%{ }^{2,3,6,7,9,13-21}$ The wide range of complication rates reported is likely due to variability in patient demographics, underlying ocular pathology, surgical technique (including type and size of implant, wrapping material, and presence or absence of sclerotomies), and duration of follow-up (with longer follow-up being associated with a higher incidence of complications). ${ }^{6,9,13,14,16,21}$ Collectively, recent reports have an average incidence of $8.6 \%$ for implant exposure, $1.0 \%$ for surgical site infection, and $2.5 \%$ for superior sulcus deformity. $2,3,6,7,9,13-21$ Our study found that primary resident cases had a below-average incidence of complications. There were no superior sulcus defects or enophthalmos noted in our study. This is likely due to follow-up of less than 5 years in the majority of resident cases, and longer follow-up may reveal the development of sulcus or socket deformities. Similar to published reports, our study did find complication rates directly correlated to the length of follow-up. Of note, the power of our study to compare resident to attending outcomes is low ( $\sim 60 \%$, confidence interval $[\mathrm{CI}]=95 \%$ ), and expanding the number of cases reviewed in the future will increase the applicability of our findings.

Minor and surgical sequelae occurred at nearly equal rates between resident-led and resident-assist surgeries. Of note, our rate of these minor and surgical sequelae seems higher than previously published reports. In the current work, $10.5 \%$ 
of resident primary cases exhibited eyelid malposition (entropion, ectropion, or blepharoptosis), and 8.8\% developed conjunctival cysts. Similarly, 10.6 and $8.5 \%$ of residentassist cases displayed similar findings, respectively. Published data show an average incidence of $7.4 \%$ for eyelid malposition and $2.2 \%$ for conjunctival cysts. ${ }^{7,9,14,16,17,19,21}$ Not all reports include these sequelae in their follow-up, and it is possible that current publications do not accurately represent the overall incidence of these sequelae. It is also possible that variations in surgical technique, underlying pathology, or presence of trauma account for the discrepancy, or that our sample is too small to reveal representative rates.

Our work is the first to propose that not only can resident surgeons safely perform ocular evisceration, but also that evisceration outcomes can be used as a marker for evaluating resident performance. For example, three of the five conjunctival cysts in resident primary cases occurred in cases performed by the same resident. Conjunctival cysts occur from erroneous implantation of conjunctiva in posterior layers like Tenon's capsule. These cases had no history of ocular trauma; as such, the occurrence of conjunctival cysts in these patients is likely a direct reflection of surgical technique and can be used to assess resident surgical performance. It is acknowledged that this assessment has more utility for short-term complications when surgical assessment and teaching occurs during residency.

This study is also the first to demonstrate that complications in resident evisceration cases are inversely correlated with the number of months the resident spent on the oculoplastic service. While a nonsignificant trend in this study, this finding is consistent with previously published work in ophthalmology surgical training. Surgical trainingincluding length of training-predicts performance and outcomes, though not necessarily transferability, across subspecialties. $^{28-34}$

Limitations of this study include the small number of residents assessed $(n=12)$ and differences in oculoplastic training. Early in the years of this retrospective chart review, residents did not have an immersive oculoplastic training period. Later, residents underwent an immersive 3-monthlong oculoplastic rotation. This allowed for better evaluation of skills and interpretation of those skills in patient outcomes. Some residents continued to perform oculoplastic cases after completion of their 3-month block, and those with contiguous months of oculoplastic performance were included in this study's data analysis. Those without a known block of contiguous months of oculoplastic training were omitted from those portions of the data analysis. This results in less statistical power, and is a source of improvement in future work. Several tools have been developed to measure resident physician technical skills, ${ }^{35-37}$ including a proposed curriculum for oculoplastic training, ${ }^{38}$ and perhaps ocular eviscerations should be considered as a safe, formalized teaching and evaluation tool for resident surgical skills.

Future directions would also include increasing the sample size and duration of follow-up studied. With a confidence interval of $5 \%$, the current work gives us a power of approxi- mately $60 \%$ when comparing resident to attending outcomes. A larger sample size is needed to improve the power of this work. In addition, while some patients were followed up up to 5 years, the majority have been followed up only for approximately 2 years to date. This is insufficient time to see complications like superior sulcus deformity and enophthalmos. Thus, larger studies with longer follow-up and an increased number of resident surgeons may be necessary to further support the findings of this study.

The goals of this project were multiple: (1) to compare resident evisceration outcomes to those in published reports; (2) to assess outcomes after ocular evisceration; and (3) to look for correlation between surgical outcomes and duration of resident's oculoplastic training. This study supports the ACGME goal of enhancing resident physician education in surgical competency, assessment of resident performance, and utilizing outcomes for improving resident education. $^{39}$ This study shows that not only are ocular eviscerations safe for resident surgeons to perform, but they are also a procedure that is ideal for training and evaluating resident surgical performance.

\section{Funding \\ None.}

\section{Conflict of Interest}

None declared.

\section{Acknowledgments}

The authors would like to thank Xiaoning Isaac Guo and SivaDurga Thotapalli for their assistance with statistical analysis.

\section{References}

1 Dortzbach RK, Woog JJ. Choice of procedure. Enucleation, evisceration, or prosthetic fitting over globes. Ophthalmology 1985; 92(09):1249-1255

2 Kostick DA, Linberg JV. Evisceration with hydroxyapatite implant. Surgical technique and review of 31 case reports. Ophthalmology 1995;102(10):1542-1548, discussion 1548-1549

3 Huang D, Yu Y, Lu R, Yang H, Cai J. A modified evisceration technique with scleral quadrisection and porous polyethylene implantation. Am J Ophthalmol 2009;147(05):924-928, 928.e1928.e3

4 Yousuf SJ, Jones LS, Kidwell ED Jr. Enucleation and evisceration: 20 years of experience. Orbit 2012;31(04):211-215

5 McAlinden C, Saldanha M, Laws D. Evisceration for the management of ocular trauma. BMJ Case Rep 2013;2013:bcr2013201235

6 Lin CW, Liao SL. Long-term complications of different porous orbital implants: a 21-year review. Br J Ophthalmol 2017;101 (05):681-685

7 Nakra T, Simon GJ, Douglas RS, Schwarcz RM, McCann JD, Goldberg RA. Comparing outcomes of enucleation and evisceration. Ophthalmology 2006;113(12):2270-2275

8 Gürdal C, Erdener U, Irkeç M, Orhan M. Incidence of sympathetic ophthalmia after penetrating eye injury and choice of treatment. Ocul Immunol Inflamm 2002;10(03):223-227

9 Blaydon SM, Shepler TR, Neuhaus RW, White WL, Shore JW. The porous polyethylene (Medpor) spherical orbital implant: a retrospective study of 136 cases. Ophthal Plast Reconstr Surg 2003;19 (05):364-371 
10 Levine MR, Pou CR, Lash RH. The 1998 Wendell Hughes Lecture. Evisceration: is sympathetic ophthalmia a concern in the new millennium? Ophthal Plast Reconstr Surg 1999;15(01):4-8

11 Hansen AB, Petersen C, Heegaard S, Prause JU. Review of 1028 bulbar eviscerations and enucleations. Changes in aetiology and frequency over a 20-year period. Acta Ophthalmol Scand 1999;77 (03):331-335

12 Shah RD, Singa RM, Aakalu VK, Setabutr P. Evisceration and enucleation: a national survey of practice patterns in the United States. Ophthalmic Surg Lasers Imaging 2012;43(05): 425-430

13 Custer PL, Trinkaus KM. Porous implant exposure: incidence, management, and morbidity. Ophthal Plast Reconstr Surg 2007; 23(01):1-7

14 Alwitry A, West S, King J, Foss AJ, Abercrombie LC. Long-term follow-up of porous polyethylene spherical implants after enucleation and evisceration. Ophthal Plast Reconstr Surg 2007;23 (01):11-15

15 Masdottir S, Sahlin S. Patient satisfaction and results after evisceration with a split-sclera technique. Orbit 2007;26(04):241-247

16 Smith RJ, Prazeres S, Fauquier S, Malet T. Complications of two scleral flaps evisceration technique: analysis of 201 procedures. Ophthal Plast Reconstr Surg 2011;27(04):227-231

17 Jung SK, Cho WK, Paik JS, Yang SW. Long-term surgical outcomes of porous polyethylene orbital implants: a review of 314 cases. Br J Ophthalmol 2012;96(04):494-498

18 Zhang Y, Zhang MN, Wang X, Chen XF. Removal of the eye in a tertiary care center of China: a retrospective study on 573 cases in 20 years. Int J Ophthalmol 2015;8(05):1024-1030

19 Kang MJ, Jung SK, Cho WK, Paik JS, Yang SW. Long-term surgical outcomes of the multi-purpose conical porous synthetic orbital implant. Korean J Ophthalmol 2015;29(05):294-300

20 Ababneh OH, AboTaleb EA, Abu Ameerh MA, Yousef YA. Enucleation and evisceration at a tertiary care hospital in a developing country. BMC Ophthalmol 2015;15:120-127

21 Verhoekx JSN, Rengifo Coolman A, Tse WHW, Paridaens D. A single- versus double-layered closure technique in anophthalmic surgery. Ophthal Plast Reconstr Surg 2017;33(05):329-333

22 Frost WA. What is the best method of dealing with a lost eye? BMJ 1887;1(1378):1153-1154

23 Green WR, Maumenee AE, Sanders TE, Smith ME. Sympathetic uveitis following evisceration. Trans Am Acad Ophthalmol Otolaryngol 1972;76(03):625-644

24 Lubin JR, Albert DM, Weinstein M. Sixty-five years of sympathetic ophthalmia. A clinicopathologic review of 105 cases (1913-1978). Ophthalmology 1980;87(02):109-121
25 Gasch AT, Foster CS, Grosskreutz CL, Pasquale LR. Postoperative sympathetic ophthalmia. Int Ophthalmol Clin 2000;40(01): 69-84

26 Custer PL, Reistad CE. Enucleation of blind, painful eyes. Ophthal Plast Reconstr Surg 2000;16(05):326-329

27 Phan LT, Hwang TN, McCulley TJ. Evisceration in the modern age. Middle East Afr J Ophthalmol 2012;19(01):24-33

28 Le TD, Adatia FA, Lam WC. Virtual reality ophthalmic surgical simulation as a feasible training and assessment tool: results of a multicentre study. Can J Ophthalmol 2011;46(01):56-60

29 Roohipoor R, Yaseri M, Teymourpour A, Kloek C, Miller JB, Loewenstein JI. Early performance on an eye surgery simulator predicts subsequent resident surgical performance. J Surg Educ 2017;74(06):1105-1115

30 Taylor JB, Binenbaum G, Tapino P, Volpe NJ. Microsurgical lab testing is a reliable method for assessing ophthalmology residents' surgical skills. Br J Ophthalmol 2007;91(12):1691-1694

31 Khalifa YM, Bogorad D, Gibson V, Peifer J, Nussbaum J. Virtual reality in ophthalmology training. Surv Ophthalmol 2006;51(03): 259-273

32 Thomsen ASS, Kiilgaard JF, la Cour M, Brydges R, Konge L. Is there inter-procedural transfer of skills in intraocular surgery? A randomized controlled trial. Acta Ophthalmol 2017;95(08):845-851

33 Deuchler S, Ackermann H, Singh P, Kohnen T, Wagner C, Koch F. Key factors to improve the outcome of retinal reattachment surgery in proliferative vitreoretinopathy and proliferative diabetic retinopathy. J Ophthalmol 2017;2017:2323897

34 Menda SA, Driver TH, Neiman AE, Blumberg S, Naseri A, Stewart JM. Risk factors for return to the operating room after residentperformed cataract surgery. Semin Ophthalmol 2018;33(02): 210-214

35 Cremers SL, Lora AN, Ferrufino-Ponce ZK. Global rating assessment of skills in intraocular surgery (GRASIS). Ophthalmology 2005;112(10):1655-1660

36 Cremers SL, Ciolino JB, Ferrufino-Ponce ZK, Henderson BA. Objective assessment of skills in intraocular surgery (OASIS). Ophthalmology 2005;112(07):1236-1241

37 Fisher JB, Binenbaum G, Tapino P, Volpe NJ. Development and face and content validity of an eye surgical skills assessment test for ophthalmology residents. Ophthalmology 2006;113(12): 2364-2370

38 Mishra K, Mathai M, Della Rocca RC, Reddy HS. Improving resident performance in oculoplastic surgery: a new curriculum using surgical wet laboratory videos. J Surg Educ 2017;74(05):837-842

39 Swing SR. The ACGME outcome project: retrospective and prospective. Med Teach 2007;29(07):648-654 